\title{
Estudos genéticos sobre a leitegada em suínos da raça Landrace criados no Rio Grande do Sul ${ }^{1}$
}

\author{
Fernanda Fernandes Lourenço², Nelson José Laurino Dionello³, Gil Carlos Rodrigo \\ Medeiros ${ }^{4}$, Valmir Costa da Rosa ${ }^{5}$
}

\author{
${ }^{1}$ Pesquisa financiada pela CAPES. \\ 2 Mestrando do Departamento de Zootecnia-DZ/FAEM/UFPEL. Bolsista CAPES. \\ ${ }^{3}$ Departamento de Zootecnia-DZ/FAEM/UFPEL. \\ ${ }^{4}$ Departamento de Informática-DINFO/IFM/UFPEL. \\ ${ }^{5}$ Serviço de Registro Genealógico de Suínos - ABCS.
}

RESUMO - Avaliaram-se parâmetros e tendências genéticas em suínos da raça Landrace em uma amostra constituída de 927 avós, 2.537 mães e 8.887 leitegadas registradas nos arquivos de dados da Associação Brasileira de Criadores de Suínos (ABCS), no estado do Rio Grande do Sul. As estimativas dos componentes genéticos foram obtidas pelo Método REML utilizando-se um modelo que incluiu os efeitos genéticos diretos e maternos; os efeitos comuns de leitegada; os efeitos fixos de grupo contemporâneo (granja, ano de nascimento dos leitões e estação do ano); e as co-variáveis número de partos da mãe e idades da mãe ao primeiro e ao último parto. As tendências genéticas diretas e maternas foram avaliadas por meio da regressão dos valores genéticos diretos e maternos sobre o ano de nascimento da mãe. As herdabilidades genéticas aditivas e maternas foram, respectivamente, de 0,0018 e 0,0117 para número de leitões ao nascimento (NLN), 0,0031 e 0,0088 para número de leitões aos 21 dias (NLD), 0,0237 e 0,0132 para peso médio ao nascimento (PMNasc), 0,0002 e 0,0081 peso médio ajustado para os 21 dias (PMD), 0,0000 e 0,0070 para ganho médio diário (GMD) e 0,0280 e 0,0103 para natimortos (NatMort). A análise de variância para as tendências genéticas, tanto para os efeitos direto quanto para o efeito materno, foi significativa para NLN, NLD, PMNasc e NatMort e não-significativa para PMD e GMD, respectivamente, considerando os dois efeitos estudados. Tendências genéticas aditivas diretas e maternas foram positivas para número de leitões ao nascimento (0,0007/ 0,0018 g/ano), número de leitões aos 21 dias (0,001/ 0,0017 g/ano) e ganho médio diário (5E-06/ 0,0003 g/ano) e negativas para peso médio ao nascimento (-0,0001/ -8E-05 g/ano), peso médio ao desmame (-1E-05/ -7E-05 g/ano) e natimortos (-0,002/ -1E-05 g/ano).

Palavras-chave: efeito genético direto e materno, pesos de leitões, tamanho de leitegada, tendências genéticas

\section{Genetic characterization of litter traits in purebred Landrace swine, in the state of Rio Grande do Sul, Brazil}

\begin{abstract}
The goals of this research were to obtain genetic parameters and estimate genetic trends in Landrace pigs. Data consisted of records from 927 granddams, 2,537 dams and 8,887 litters raised in the state of Rio Grande do Sul, Brazil, provided by the Swine Breeders Brazilian Association - ABCS. Variance components were estimated by REML, using a model that included the direct genetic effect, maternal effect, common litter effect and the fixed effect of contemporary group. Contemporary groups were defined as a combination of farm, year, and season of farrowing. The model also included the covariates parity order, age of sow at first litter and age of sow at last litter. Direct genetic and maternal genetic trends were estimated by regression of direct genetic values on birth year of the dam. Heritability and maternal heritabilities were, respectively, .002 and .012 for litter size at birth (NLN), .003 and .009 for litter size at 21 days (NLD), .024 and .013 for average litter weight at birth (PMNasc), .000 and .008 for average litter weight at weaning, in kg, adjusted to 21 days (PMD), .000 and .007 for average daily weight gain (GMD), .028 and .010 for number of stillborn (NatMort). Analysis of variance for genetic trend showed significance for direct and maternal genetic effects for NLN, NLD, PMNasc and NatMort but not for PMD or GMD. Genetic trends were positive for NLN (0.0007/ 0.0018 g/year), NLD (0.001/ 0.0017 g/year), GMD (5E-06/ 0.0003 g/year) and negative for (-0.0001/ -8E-05 g/year), PMD (-1E-05/ -7E-05 g/year) e NatMort (-0.002/ -1E-05 g/year).
\end{abstract}

Key Words: direct and maternal genetic effect, genetic trend, litter size, piglet weight

Este artigo foi recebido em 27/3/2007 e aprovado em 26/3/2008.

Correspondências devem ser enviadas parafeloma@ig.com.br. 


\section{Introdução}

A raça Landrace foi desenvolvida na Dinamarca a partir do cruzamento do suíno nativo com o da raça Large White. Esse cruzamento foi melhorado durante anos sob controle rígido do governo; sua criação visava à produção de toucinho e um animal adaptado ao sistema intensivo de criação. Atualmente, é a segunda raça mais popular de suínos (Taylor et al., 2005) e com aptidão predominante da raça para produção de carne. Para atender essa produção, são produzidos animais precoces, com fecundidade boa: 10 a 12 leitões possuem crescimento rápido (FZEA-USP, 2005) e peso ao desmame superior ao de outras raças. Além disso, é uma raça que produz pernil longo e capaz de produzir excelentes presuntos (Taylor et al., 2005).

A raça Landrace em 1998 participou com 15,47\% dos registros puros de origem emitidos no País, portanto, foi a segunda raça com maior número de animais registrados (NESUI, 2005). Ocupou em 2005 o $3^{\circ}$ lugar em número de reprodutores, vindo logo depois do Duroc e Wessex. Os melhores cruzamentos para produção de carne foram obtidos com o uso de cachaços Landrace e de fêmeas Duroc, Wessex e mestiças dessas duas raças (FZEA-USP, 2005).

Em virtude das características promissoras da raça, o melhoramento animal tem por finalidade aperfeiçoar a produção. Para que isso ocorra, deve-se definir uma estratégia de seleção visando maiores respostas genéticas no menor intervalo de tempo (Ramalho et al., 1989). A renda que esse animal gera para o País com as exportações de carne cresceu 81,11\% nos cinco primeiros meses de 2006 em relação ao mesmo período do ano anterior (Quevedo, 2005); a carne suína, em abril de 2004, aumentou 96,3\% em relação a abril de 2003 atingindo receita em torno de US\$ 53,3 milhões, segundo Roppa (2004), o que comprova a importância desses fatores.

Como o progresso genético dessas características de desempenho em uma população de suínos depende dos ganhos genéticos obtidos nos núcleos de seleção, é fundamental a avaliação periódica da eficiência do programa de melhoramento genético utilizado nestes núcleos (Pita \& Albuquerque, 2001).

Avaliaram-se neste estudo os parâmetros genéticos das características de leitegada e as tendências genéticas em suínos da raça Landrace registrados na ABCS criados no Rio Grande do Sul.

\section{Material e Métodos}

Este trabalho foi realizado no Departamento de Zootecnia da Faculdade de Agronomia Eliseu Maciel da Universidade
Federal de Pelotas. O estudo foi baseado em informações contidas nos arquivos de dados da raça Landrace da Associação Brasileira de Criadores de Suínos (ABCS) no estado do Rio Grande do Sul. As informações totalizavam 927 avós, 2.537 mães e 8.887 leitegadas da raça Landrace pura, distribuídas em 28 granjas do estado no período de 1990 a 2002. As mães e as avós deveriam ter no mínimo duas leitegadas registradas no arquivo.

Na análise, calculou-se e utilizou-se o peso médio do leitão, uma vez que no arquivo era descrito unicamente o peso da leitegada (muito variável de acordo com o número de leitões nascidos). A partir desse cálculo, foram excluídos do banco de dados os pesos médios do leitão ao nascer inferiores a $700 \mathrm{~g}$, superiores a $3.500 \mathrm{~g}$ ou cujo valor médio diário fosse negativo.

Todas as datas foram separadas do ano e transformadas em data juliana para melhor interpretação dos programas. $\mathrm{O}$ ano foi dividido em quatro estações de parição: verão - dezembro a fevereiro; outono - março a maio; inverno - junho a agosto; primavera - setembro a novembro. As características avaliadas foram: peso ao nascer (PMNasc), peso médio ajustado para 21 dias (PMD), ganho médio diário (GMD), número de leitões nascidos (NLN) e número de leitões aos 21 dias (NLD), que representam o mérito genético aditivo das mães. As padronizações dos pesos às idades consideradas foram feitas pelo método matemático de interpolação linear, ou seja, em um gráfico faz-se uma reta com os pesos e as idades registradas na associação e estima-se o peso aos 21 dias padronizando os dados.

Para todas as estimativas das características analisadas, utilizou-se um modelo incluindo os efeitos genéticos, diretos e maternos e comuns de leitegada e, como efeitos fixos, o grupo contemporâneo (granja, ano de nascimento dos leitões e estação do ano) e as co-variáveis número de partos da mãe, idade da mãe ao primeiro parto e idade da mãe ao último parto.

As estimações de componentes de variância para características reprodutivas e os parâmetros genéticos foram calculados pelo método de máxima verossimilhança restrita (REML) utilizando-se o algoritmo não-derivativo DFREML - Derivative Free Restricted Maximum Likelihood (Meyer, 1988) em equações de modelo animal uni-característico pelo DFUNI com critério de convergência inferior a $10^{-8}$.

O modelo utilizado nas análises foi:

$$
\mathrm{y}=\mathrm{Xb}+\mathrm{Z}_{1} \mathrm{~d}+\mathrm{Z}_{2} \mathrm{~m}+\mathrm{Z}_{3} \mathrm{p}+\mathrm{e},
$$

em que $\mathrm{y}$ = vetor de observações; $\mathrm{X}=$ matriz de incidência de efeitos fixos; $b$ = vetor de efeitos fixos; $Z_{1}=$ matriz de incidência de efeitos genéticos aditivos diretos; $d$ = vetor de efeitos genéticos aditivos diretos; $Z_{2}=$ matriz de incidência de afeitos genéticos aditivos maternos; $\mathrm{m}$ = vetor de 
efeitos genéticos aditivos maternos; $\mathrm{Z}_{3}=$ matriz de incidência de efeitos comuns de leitegada; $\mathrm{p}=$ vetor de efeitos comuns de leitegada; e = vetor de efeito residual, no qual o efeito direto é atribuído à porca e o materno à mãe da porca (avó dos leitões) e são considerados correlacionados.

\section{Resultados e Discussão}

Considerando as médias e os desvios-padrão, mínimo e máximo das características estudadas (Tabela 1), as médias das características mantiveram-se dentro dos parâmetros esperados para suínos, descritos em várias pesquisas anteriores, exceto a característica natimortos, que foi $50 \%$ abaixo da encontrada por Wilson et al. (1986) e Stein et al. (1990), de 0,8 e 1,0, respectivamente.

As estimativas de componentes de variância e de co-variância e dos parâmetros genéticos obtidas nas análises realizadas por meio do DFUNI (DFREML) foram inferiores às variâncias genéticas aditivas diretas e maternas (Tabela 2) encontradas por Pires et al. (2000b) para as características NLN, NLD, PMNasc e PMD e às encontradas por Chen et al. (2003) para as características NLN e NLD para animais desta raça.
Ferraz Filho et al. (2002), na obtenção de estimativas de herdabilidade para pesos corporais em populações de zebuínos, encontraram grandes variações entre os valores obtidos e os citados na literatura e comentaram que os valores destas estimativas variam de acordo com a composição genética da população e com as condições do ambiente. Magnabosco et al. (2005), em outro trabalho com zebuínos utilizando o DFREML, atribuíram o fato de os valores genéticos terem sido menores que os revisados no ano de realização dos trabalhos, uma vez que a metodologia disponível apresentava propriedades diferentes da atualmente utilizada.

Nas Figuras 1 a 6, constam os gráficos das tendências genéticas diretas e maternas na seguinte ordem de características: NLN, NLD, NatMort, PMNasc, PMD e GMD. As tendências estão ordenadas por diferença esperada por progênie (Dep) de acordo com o ano de nascimento das porcas (mães).

As características de pesos corporais apresentaram tendências negativas ao longo dos anos, enquanto as características de ganhos de peso não tiveram tendências definidas, evidenciando estagnação da população. As características relacionadas a número de leitões (NLN e

Tabela 1 - Número de registros, médias, desvios-padrão e amplitudes observadas para as características

\begin{tabular}{|c|c|c|c|c|c|c|}
\hline Característica* & Unidade & № de registros & Média & $\mathrm{DP}$ & Mínimo & Máximo \\
\hline NLN & $\mathrm{n}$ & 8.887 & 9,96 & 2,54 & 1,00 & 19,00 \\
\hline NLD & $\mathrm{n}$ & 8.887 & 9,73 & 2,51 & 1,00 & 19,00 \\
\hline PMD & $\mathrm{kg}$ & 8.887 & 6,02 & 0,98 & 3,50 & 9,46 \\
\hline GMD & g & 8.887 & 212,52 & 47,81 & 57,00 & 385,00 \\
\hline NatMort & $\mathrm{n}$ & 8.887 & 0,40 & 0,87 & 0,00 & 14,00 \\
\hline
\end{tabular}

* NLN = número de leitões nascidos; NLD = número de leitões aos 21 dias; PMNasc = peso médio da leitegada ao nascimento; PMD = peso médio ajustado para os 21 dias; GMD = ganho médio diário; Natmort = número de leitões nascidos mortos.

Tabela 2 - Estimativas dos componentes de co-variância e parâmetros genéticos obtidos por meio de análise unicaracterística

\begin{tabular}{|c|c|c|c|c|c|c|}
\hline \multirow[t]{2}{*}{ Estimativa* } & \multicolumn{6}{|c|}{ Característica } \\
\hline & NLN & NLD & PMNasc & PMD & GMD & NatMort \\
\hline$\sigma_{\mathrm{d}}^{2}$ & 0,0108 & 0,0175 & 0,0010 & 0,0001 & 0,0029 & 0,0186 \\
\hline$\sigma_{\mathrm{m}}^{2}$ & 0,0694 & 0,0505 & 0,0005 & 0,0058 & 10,9656 & 0,0068 \\
\hline$\sigma_{\mathrm{dm}}$ & 0,0274 & 0,0297 & 0,0002 & 0,0009 & 0,1690 & $-0,0044$ \\
\hline$\sigma_{p}^{2}$ & 0,6444 & 0,5866 & 0,0065 & 0,0460 & 84,7244 & 0,0109 \\
\hline$\sigma_{e}^{2}$ & 5,2059 & 5,0471 & 0,0326 & 0,6613 & $1.469,6343$ & 0,6306 \\
\hline$\sigma_{f}^{2}$ & 5,9580 & 5,7314 & 0,4080 & 0,7141 & $1.565,4962$ & 0,6624 \\
\hline$h^{2}{ }_{d}$ & 0,0018 & 0,0031 & 0,0237 & 0,0002 & 0,0000 & 0,0280 \\
\hline $\mathrm{h}^{2}{ }_{\mathrm{m}}^{\mathrm{u}}$ & 0,0117 & 0,0088 & 0,0132 & 0,0081 & 0,0070 & 0,0103 \\
\hline $\mathrm{r}_{\mathrm{dm}}$ & 1,0000 & 1,0000 & 0,3100 & 0,9976 & 0,9415 & $-0,3939$ \\
\hline $\mathrm{h}^{2}{ }_{\mathrm{t}}$ & 0,0145 & 0,0152 & 0,0039 & 0,0061 & 0,0037 & 0,0231 \\
\hline$c^{2}$ & 0,1082 & 0,1023 & 0,1594 & 0,0644 & 0,0541 & 0,0165 \\
\hline
\end{tabular}

* $\sigma^{2}{ }_{d}=$ variâncias genéticas aditivas diretas, $\sigma_{m}^{2}=$ variância genética aditiva materna, $\sigma_{d m}=$ co-variância genética entre os efeitos aditivos direto e materno $\sigma_{p}^{2}=$ variância atribuida aos efeitos comuns de leitegada, $\sigma_{e}^{2}=$ variância residual, $\sigma_{f}^{2}=$ variância fenotípica, $h^{2}=$ herdabilidade genética aditiva direta, $\mathrm{h}^{2}{ }_{\mathrm{m}}$ herdabilidade aditiva materna, $\mathrm{r}_{\mathrm{gm}}=$ correlação entre efeitos aditivos direto e materno, $\mathrm{h}^{2} \mathrm{t}=$ herdabilidade total (total heritability), $\mathrm{c}^{2}=$ proporção atribuída aos efeitos comuns de leitegada. 


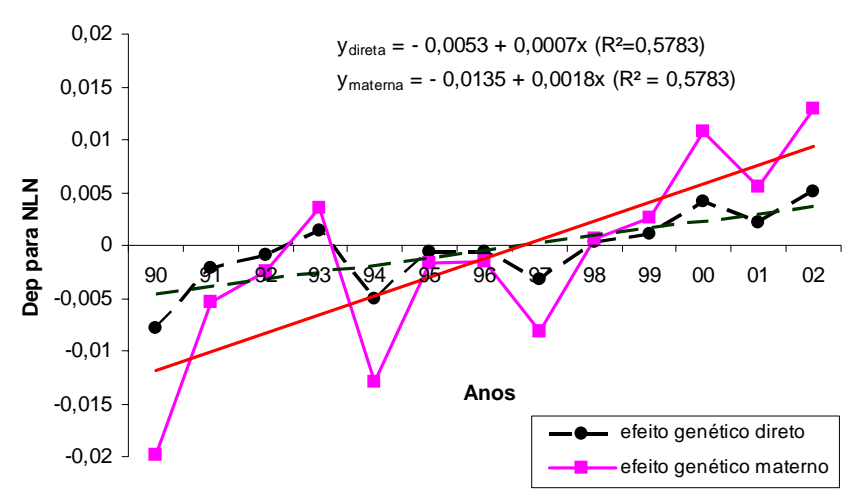

Figura 1 - Tendência dos efeitos genéticos diretos e maternos para número de leitões nascidos (NLN) no período de 1990 a 2002.

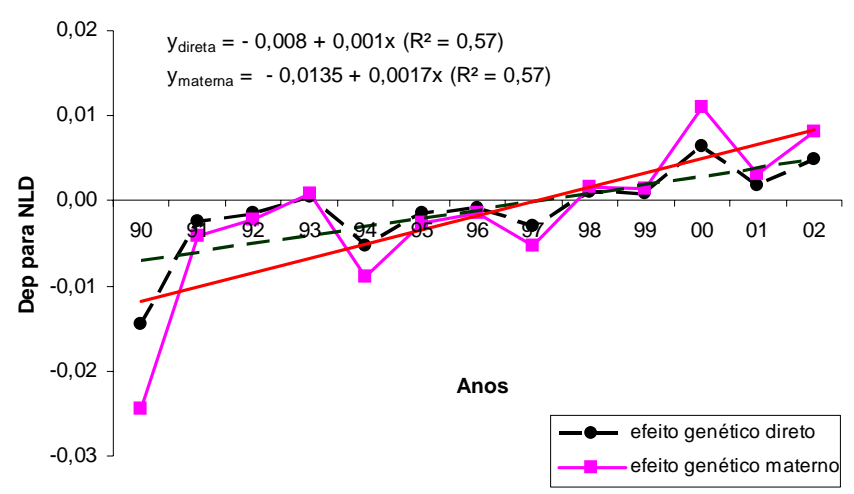

Figura 2 - Tendência dos efeitos genéticos diretos e maternos para número de leitões aos 21 dias (NLD) no período de 1990 a 2002.

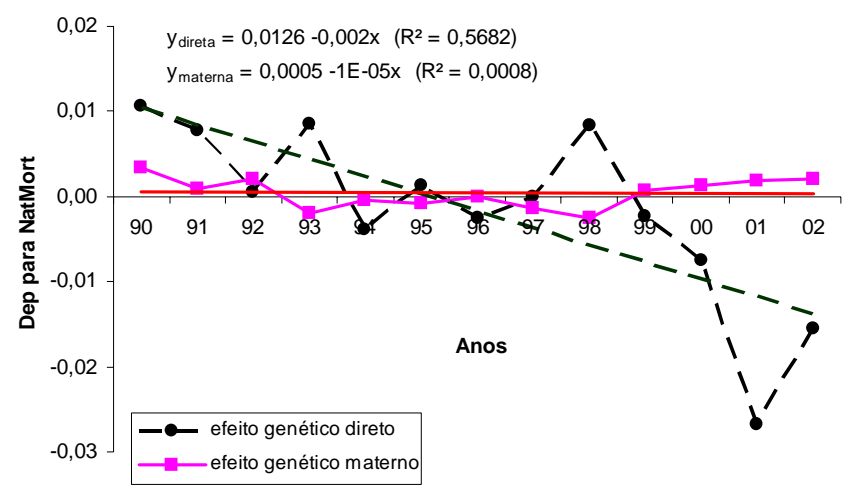

Figura 3 - Tendência dos efeitos genéticos diretos para número de leitões nascidos mortos (NatMort) no período de 1990 a 2002.

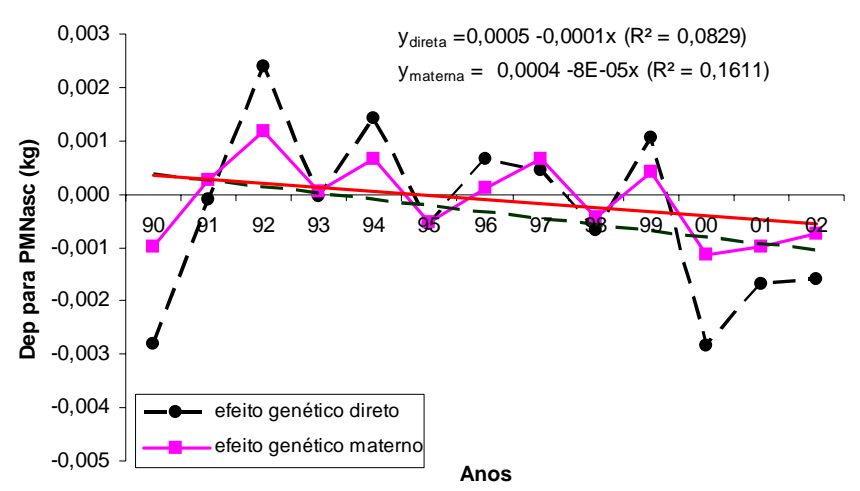

Figura 4 - Tendência dos efeitos genéticos diretos para peso médio ao nascimento (PMNasc), em kg, no período de 1990 a 2002.

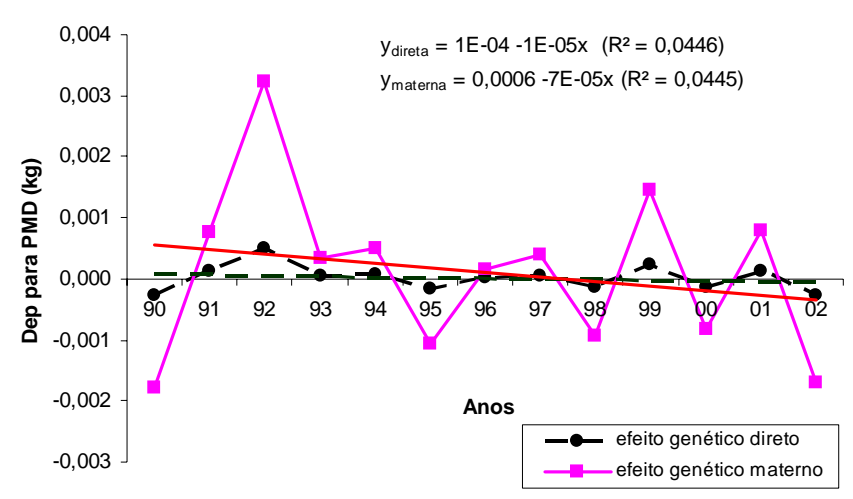

Figura 5 - Tendência dos efeitos genéticos diretos para peso de leitegada ao desmame, ajustado para 21 dias (PMD), em kg, no período de 1990 a 2002.

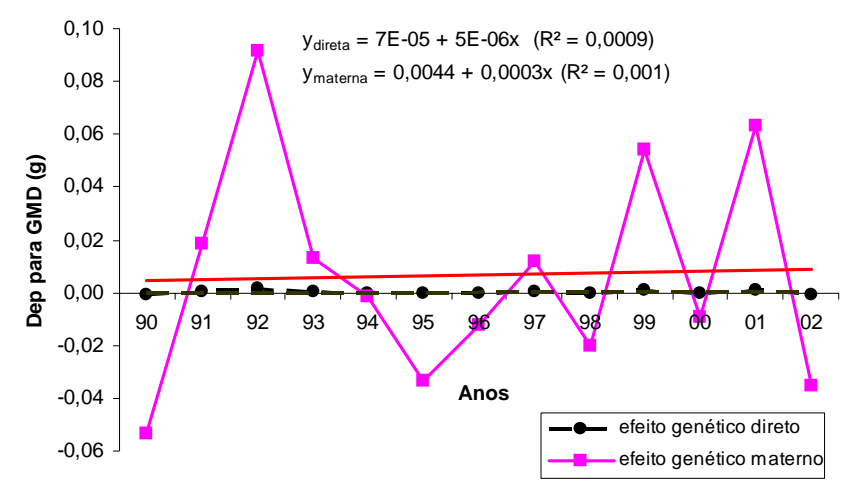

Figura 6 - Tendência dos efeitos genéticos diretos para ganho médio diário de peso de leitegada (GMD), em g, no período de 1990 a 2002.

(c) 2008 Sociedade Brasileira de Zootecnia 
NLD) apresentaram tendências positivas, mostrando a presença de animais melhoradores na população e que podem ser melhor utilizados pelos produtores. Na verdade, estas características apresentam antagonismo, pois, ao aumentar o número de leitões, em virtude da capacidade uterina da porca, houve redução no peso dos leitões. Esses resultados para as características de número de animais são diferentes dos obtidos por Pires et al. (2000a).

Apesar dos pequenos ganhos ao longo dos 12 anos estudados, a ocorrência de ganhos possibilita que os produtores tenham condições de fazer um direcionamento visando à adoção de um programa de melhoramento genético com máxima rapidez, especialmente para características em que o efeito genético materno está regredindo ou mesmo estagnado. Resultados semelhantes foram constatados por Pires et al. (2000a), que verificaram a necessidade de redirecionamentos nos programas de melhoramento utilizados para suínos ou da possível discordância entre a escolha das características (número ou desempenho) entre as granjas estudadas.

Ressalta-se que as características número de leitões e peso de leitegada no período até 21 dias apresentam baixas herdabilidades (5 a 15), como observado por Cardellino \& Rovira (1987), o que dificulta o processo de ganhos genéticos para essas características. Igualmente, Irgang et al. (1992) também verificaram pouca expressão do ganho genético para as características de leitões nascidos vivos.

Constata-se, pelas estimativas genéticas médias anuais (Tabela 3), que, para as características PMD e GMD, não houve efeitos $(\mathrm{P}>0,001)$ genéticos direto e materno. Neste caso, pode-se atribuir a falta de significância à grande influência ambiental sobre estas duas características, uma vez que os baixos efeitos genéticos e maternos indicam que pode não estar ocorrendo seleção para estas características.

A raça Landrace sempre foi considerada raça materna, portanto, deve-se dar maior atenção ao critério de seleção para as características reprodutivas (tamanho e peso das leitegadas e natimortalidade), a fim de se obter maiores ganhos em sua produtividade. Assim, o efeito genético materno, mesmo de valores mais baixos, influencia as características incluídas neste trabalho e sua não inclusão nos modelos estudados, segundo Roehe \& Kennedy (1993), tem como conseqüência a obtenção de tendências genéticas viesadas.

Tabela 3 - Tendências genéticas anuais e respectivos desvios-padrão (entre parênteses) e significâncias para efeito genético direto e materno

\begin{tabular}{lcrrr}
\hline Característica & Efeito genético direto & F & Efeito genético materno & F \\
\hline NLN & $0,0007(0,021)$ & $<0,001$ & $0,0018(0,052)$ & $<0,001$ \\
NLD & $0,001(0,027)$ & $<0,001$ & $0,0017(0,045)$ & $<0,001$ \\
NatMort & $-0,002(0,044)$ & $<0,001$ & $0,00001(0,013)$ & $<0,001$ \\
PMNasc & $-0,0001(0,009)$ & $<0,001$ & $-0,00008(0,005)$ & 0,0001 \\
PMD & $-0,00001(0,002)$ & 0,015 & $0,0003(0,011)$ & $0,085)$ \\
GMD & $0,000005(0,006)$ & 0,223 & 0,226 \\
\hline
\end{tabular}

NLN = número de leitões nascidos; NLD = número de leitões aos 21 dias; Natmort = número de leitões nascidos mortos; PMNasc = peso médio da leitegada ao nascimento; PMD = peso médio da leitegada ao 21 dias; GMD = ganho médio diário.

\section{Conclusões}

Existem animais melhoradores na população para as características número de leitões nascidos e desmamados e natimortos. Entretanto, a população encontra-se em regressão para a característica peso médio ao nascimento e está estagnada para as características peso médio ao desmame e ganho médio diário. Para que não seja perdida a genética materna, é necessária a utilização de um programa de melhoramento genético pelos produtores.

\section{Agradecimento}

A Marcella Bicca Bragança Correa, por seu apoio na realização deste trabalho. Aos meus familiares, especialmente à minha mãe e a André Borba Affonso.

\section{Literatura Citada}

CARDELLINO, R.A.; ROVIRA, J. Mejoramiento genetico animal. Montevideo: Editorial Hemisferio Sur, 1987. 253p.

CHEN, P.; BAAS, T.J.; MABRY, J.W. et al. Genetic parameters and trends for litter traits in U.S. Yorkshire, Duroc, Hampshire, and Landrace pigs. Journal of Animal Science, v.81, p.4653, 2003.

FERRAZ FILHO, P.B.; RAMOS, A.A.; SILVA, L.O.C. et al. Herdabilidade e correlações genéticas, fenotípicas e ambientais para pesos em diferentes idades de bovinos da raça Tabapuã. Archives of Veterinary Science, v.7, n.1, p.65-69, 2002.

FZEA-USP, EQUIPE DE MELHORAMENTO GENÉTICO. Raças. Disponível em: http://criareplantar.com.br/pecuaria/suino/ zootecnia.php?tipoConteudo $=$ texto\&idConteudo $=126$. Acesso em: 19/12/2005.

IRGANG, R.; FAVERO, J.A.; SCHEID, I. Heterose e complementariedade entre raças na produção de suínos para o abate. Suinocultura Dinâmica, Ano I, n.3, 6p, 1992.

MAGNABOSCO, C.U.; FAMULA, T.R.; LOBO, R.B. et al. "Estimativas de parâmetros genéticos e de ambiente de características de crescimento em bovinos da raça Nelore”. Disponível em: 
http://www.sbz.org.br/eventos/Fortaleza/Melh_anim\% 5CSbz067.pdf. Acesso em: 29/11/2005.

MEYER, K. DFREML - Programs to estimate variance components for individual animal models by restricted maximum likelihood. United Kingdom: University of Edinburgh, 1988.

NÚCLEO DE ESTUDO EM SUINOCULTURA - NESUI. Raças Disponível em: http://www.nucleoestudo.ufla.br/nesui/racas.htm. Acesso em: 19/12/2005.

PIRES, A.V.; LOPES, P.S.; TORRES, R.A. et al. Tendências genéticas dos efeitos genéticos direto e materno em características reprodutivas de suínos. Revista Brasileira de Zootecnia, v.29, n.6, p.1689-1697, 2000a.

PIRES, A.V.; LOPES, P.S.; TORRES, R.A. et al. Estimação de parâmetros genéticos de características reprodutivas em suínos. Revista Brasileira de Zootecnia, v.29, n.6, p.1698-1705, 2000 b.

PITA, F.V.C.; ALBUQUERQUE, L.G. Resposta à seleção para características de desempenho em um rebanho de seleção de suínos. Revista Brasileira de Zootecnia, v.30, n.6, p.2009-2016, 2001 (supl.).

QUEVEDO, A.C. Exportações brasileiras de carne suína. Revista Suinocultura Industrial, v.187, n.4, p.25, 2005.
RAMALHO, M.A.P.; SANTOS J.B.; PINTO, C.B. Genética na agropecuária. São Paulo: Globo/ Fundação de Apoio ao Ensino, Pesquisa e Extensão, 1989. 359p.

ROEHE, R.; KENNEDY, B.W. Efficiency of an approximate animal model for maternal and diretic effects of litter size in swine. Journal of Animal Science, v.71, n12, p.3251-60, 1993.

ROPPA, F. Notícias Curtas - Dados preliminares apontam incremento de $96,3 \%$ na receita obtida com exportação de carne suína no mês de abril. Revista Pork World, Ano 3, n.20, p.16, 2004.

STEIN, T.E.; DUFFY, S.J.; WICKSTROM, S. Differences in production values between highand low-productivity swine breeding herds. Journal of Animal Science, v.68, p.39723979, 1990.

TAYLOR, G.; ROESE, G.; HERMESCH, S. [2005]. Breeds of pigsLandrace. Disponível em http://www.dpi.nsw.gov.au/_data/assets/ pdf_file/45557/Breeds_of_pigs-Landrace_-_Primefact_63final.pdf. Acesso em: 19/12/2005.

WILSON, M.R.; FRIENDSHIP, R.M.; MCMILLAN, I. et al. A survey of productivity and its component interrelationship in Canadian swine herds. Journal of Animal Science, v.62, p.576-582, 1986. 